\title{
PARTICLE ACCELERATION IN SPHERICAL WAVE FIELDS
}

\author{
K. O. THIELHEIM
}

Institut für Reine und Angewandte Kernphysik, Abteilung Mathematische Physik, Universität Kiel

\section{Particle acceleration by rotat- ing magnets}

A magnet rotating in vacuo with its vector of angular velocity $\omega$ perpendicular to its vector of magnetic dipole moment $\mu$ is able to act as a particle accelerator. The dynamic features involved may be relevant for the understanding of rotating magnetized neutron stars as cosmic accelerators (Thielheim 1989) and may be useful for the designing of new mechanisms for accelerating devices.

In the present paper I will consider the acceleration of particles initially at rest, from positions between the acceleration boundary

$$
r_{\mathrm{B}} \cong r_{\mathrm{T}}\left(r_{\mathrm{T}} / r_{\mathrm{L}}\right)^{1 / 3}
$$

and the plasma border

$$
r_{\mathrm{P}} \cong\left(2^{-1 / 2}\right)(\beta \gamma)^{-1} r_{\mathrm{T}}\left(r_{\mathrm{T}} / r_{\mathrm{L}}\right)
$$

within the wave zone of a rotating magnet. By definition the 'light radius'

$$
r_{\mathrm{L}}=c / \omega
$$

will be used as a unit of length throughout this paper, so that all coordinates are written in dimensionless form. The 'typical radius'

$$
r_{\mathrm{T}}=\left(e \mu / m c^{2}\right)^{1 / 2}
$$

also with the dimension of a length, will be used to characterize the electromagnetic properties of the system at hand.

Special attention will be paid to the mean final energy of such particles and to the resulting differential energy spectrum as obtained on the basis of given source functions within the range $r_{\mathrm{B}}<r_{0}<r_{\mathrm{P}}$ (Thielheim and Tolan 1990).

\section{Vacuum wave fields of a rotat- ing magnet}

Since particle dynamics under given premises are essentially determined by the spherical character of the wave field, it is convenient to use spherical coordinates $x^{1}=r, x^{2}=\theta, x^{3}=\phi$ instead of cartesian coordinates $x=r \sin \theta \cos \phi, y=r \sin \theta \sin \phi, z=$ $r \cos \theta$.

The mixed components of the field tensor defined with reference to these spherical coordinates are also given in dimensionless form by

$$
\left(F_{k}^{i}\right)=\frac{r_{\mathrm{T}}^{2}}{r_{\mathrm{L}}^{2}}\left(\begin{array}{rrrr}
0 & 0 & a & -b \\
0 & 0 & a & -b \\
a / r^{2} & -a / r^{2} & 0 & 0 \\
-q / r^{2} & q / r^{2} & 0 & 0
\end{array}\right),
$$

with $a=\sin \Phi, b=\sin \theta \cos \theta \cos \Phi, q=\cot \theta \cos \Phi$ and $\Phi=\left(x^{0}-x^{1}\right)-\phi$.

\section{Equations of motion}

The Lorentz equation for arbitrary coordinates

$$
\frac{D u^{i}}{d s}=F_{k}^{i} u^{k}
$$

on its left side involves the covariant derivative of the vector of velocity with respect to eigentime

$$
\frac{D u^{i}}{d s}=\frac{d u^{i}}{d s}+\Gamma_{k l}^{i} u^{k} u^{l},
$$

and therefore has to be calculated by means of the Christoffel symbols

$$
\Gamma_{k l}^{i}=g^{i m}\left\{\partial_{L} g_{k m}+\partial_{k} g_{m l}-\partial_{m} g_{k l}\right\} / 2,
$$

which in the case of spherical coordinates are given by

$$
\begin{aligned}
& \Gamma_{22}^{1}=-r ; \quad \Gamma_{33}^{1}=-r \sin ^{2} \theta \\
& \Gamma_{33}^{2}=-\sin \theta \cos \theta \\
& \Gamma_{12}^{2}=\Gamma_{21}^{2}=\Gamma_{13}^{3}=\Gamma_{31}^{3}=1 / r \\
& \Gamma_{23}^{3}=\Gamma_{32}^{3}=\cot \theta \\
& \Gamma_{k l}^{i}=0 \text { for all other indices. }
\end{aligned}
$$

The components of the vector of velocity are also written in dimensionless form, i.e. in units of the velocity of light,

$$
\begin{gathered}
u^{0}=c \frac{d t}{d s}=\gamma \\
d u^{1}=\frac{d r}{d s}=u_{r} \\
u^{2}=\frac{d \theta}{d s}=u_{\theta} / r \\
u^{3}=\frac{d \phi}{d s}=u_{\phi} / r \sin \theta
\end{gathered}
$$


where $d s$ is the dimensionless differential of eigen- for which equation (11d) reduces to time,

$$
d s=c d \tau / r_{\mathrm{L}}=c d t / \gamma r_{\mathrm{L}}
$$

$$
\frac{d u_{\phi}}{d s}=-u_{r} u_{\phi} / r
$$

so that

$$
u^{i} u_{i}=\gamma^{2}-u_{r}^{2}-u_{\theta}^{2}-u_{\phi}^{2}=1
$$

With the definition

$$
\hat{u}=r u_{\phi}
$$

Use will also be made of the definition $\alpha=\gamma-u_{r}$.

The components of the Lorentz equation then are obtained in the form

$$
\begin{gathered}
\frac{d \gamma}{d s}=\frac{r_{T}^{2}}{r_{\mathrm{L}}^{2}} \frac{u_{\theta} \sin \Phi-u_{\phi} \cos \theta \cos \Phi}{r} \\
\frac{d u_{r}}{d s}=\frac{u_{\theta}^{2}+u_{\phi}^{2}}{r}+\frac{r_{\mathrm{T}}^{2}}{r_{\mathrm{L}}^{2}} \frac{u_{\theta} \sin \theta-u_{\phi} \cos \theta \cos \Phi}{r} \\
\frac{d u_{\theta}}{d s}=\frac{u_{\phi}^{2} \cot \theta-u_{r} u_{\theta}}{r}+\frac{r_{\mathrm{T}}^{2}}{r_{\mathrm{L}}^{2}}\left(\frac{\alpha}{r}\right) \sin \Phi \\
\frac{d u_{\phi}}{d s}=-\frac{u_{\theta} u_{\phi} \cot \theta+u_{r} u_{\theta}}{r}-\frac{r_{\mathrm{T}}^{2}}{r_{\mathrm{L}}^{2}}\left(\frac{\alpha}{r}\right) \cos \theta \cos \Phi .
\end{gathered}
$$

Equations (10a) to (10d) and (11a) to (11d) constitute the equations of motion for the problem discussed here.

It is useful to note that as a consequence of the definitions given above

$$
u_{r}=\left\{1+u_{\theta}^{2}+u_{\phi}^{2}-\alpha^{2}\right\} / 2 \alpha .
$$

\section{Constant latitude approxima- tion and restriction to the equatorial plane of rotation}

The topography of orbits and the development of energy are considered for particles initially at rest and originating from positions within the range $r_{\mathrm{B}}<r_{0}<r_{\mathrm{P}}$ for parameter values $\omega$ and $\mu$, or alternatively $r_{\mathrm{L}}$ and $r_{\mathrm{T}}$, typically observed in the physics of rotating magnetized neutron stars.

Under these premises the 'constant latitude approximation'

$$
\theta(s)=\theta\left(s_{0}\right)=\theta_{0}
$$

implying that particle orbits essentially remain confined to the neighborhood of a certain locus of constant latitude $\theta_{0}$ has been found to be justified by comparison with the results of numerical iniegration.

For the present purposes it is sufficient to consider particle motion near the equatorial plane of rotation

$$
\theta_{0}=\pi / 2
$$

one is lead to

$$
\frac{d \hat{u}}{d s}=r \frac{d u_{\phi}}{d s}+u_{r} u_{\phi}
$$

which together with equation (15) is equivalent to

$$
\frac{d \hat{u}}{d s}=0 .
$$

The solution of this equation for given initial conditions is

$$
\hat{u}(s)=\hat{u}\left(s_{0}\right)=0
$$

so that

$$
u_{\phi}=0
$$

and therefore

$$
\phi=\phi\left(s_{0}\right)=\phi_{0}=\text { const. }
$$

Consequently, particle orbits under given premises not only are restricted to regions near to a conus of constant latitude, but also to regions near to a plane of constant longitude, in other words, near to a straight line pointing outward from the rotating magnet in the radial direction.

As a consequence of the 'constant-latitude approximation' one may adopt the initial value of longitude $\phi_{0}=0$, so that the phase $\Phi$ may be written in the form

$$
\Phi=x^{0}-x^{1} .
$$

Differentiation with respect to eigentime then delivers

$$
\frac{d \Phi}{d s}=\alpha .
$$

Substracting equation (11b) from equation (11a) results in

$$
\frac{d \alpha}{d s}=-u_{\theta}^{2} / r
$$

The latter equations together with

$$
\begin{gathered}
\frac{d u_{r}}{d s}=\left\{\frac{r_{T}^{2}}{r_{L}^{2}} u_{\theta} \sin \Phi+u_{\theta}^{2}\right\} / r \\
\frac{d u_{\theta}}{d s}=\frac{r_{\Gamma}^{2}}{r_{L}^{2}} \frac{\alpha}{r} \sin \Phi-u_{r} u_{\theta} / r
\end{gathered}
$$

obtained from equations (11b) and (11c) respectively, and with equations (10b) and (10c) now replace the original set of equations of motion. 


\section{Substitution of eigentime by phase}

Differentiation with respect to eigentime may be substituted with the help of equation (23) by differentiation with respect to phase,

$$
\frac{d}{d s}=\alpha \frac{d}{d \Phi}
$$

It is convenient to introduce

$$
\tilde{u}=r u_{\theta}
$$

so that

$$
\frac{d \tilde{u}}{d s}=r d u_{\theta} / d \Phi+u_{r} u_{\theta} / \alpha .
$$

Equation (26) then leads to

$$
\frac{d \tilde{u}}{d \Phi}=\left(r_{\mathrm{T}} / r_{\mathrm{L}}\right)^{2} \sin \Phi
$$

so that

$$
\tilde{u}=\tilde{u}_{0}+\left(r_{\mathrm{T}} / r_{\mathrm{L}}\right)^{2}\left(\cos \Phi_{0}-\cos \Phi\right)
$$

where $\check{u}_{0}=\tilde{u}\left(\Phi_{0}\right)=0$ according to initial conditions adopted here.

Correspondingly, equation (24) leads to

$$
\frac{d \alpha}{d \Phi}=-\tilde{u}^{2} / \alpha r^{3}
$$

while equation $(10 \mathrm{~b})$ now becomes

$$
\frac{d r}{d \Phi}=\left\{r^{2}\left(1-\alpha^{2}\right)+\tilde{u}^{2}\right\} / 2 \alpha^{2} r^{2} .
$$

The set of equations of motion thereby has been reduced to (31), (32), and (33).

\section{Mean development of dynamic variables}

Since by equation (31) $\tilde{u}^{2}$ is a periodic function of phase

$$
\tilde{u}^{2}=\left(r_{\mathrm{T}} / r_{\mathrm{L}}\right)^{4}\left(\cos \Phi_{0}-\cos \Phi\right)^{2}
$$

oscillating about a mean value

$$
\overline{\tilde{u}^{2}}=\left(r_{\mathrm{T}} / r_{\mathrm{L}}\right)^{4}\left(1+2 \cos ^{2} \Phi_{0}\right) / 2
$$

the mean development of dynamic variables is ob-

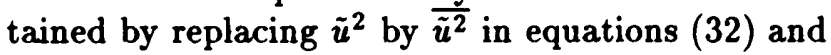
(33) so that

$$
\frac{d \alpha}{d \Phi}=-\overline{\tilde{u}^{2}} / \alpha r^{3}
$$

$$
\frac{d r}{d \Phi}=\left\{r^{2}\left(1-\alpha^{2}\right)+\overline{\tilde{u}^{2}}\right\} / 2 \alpha^{2} r^{2} .
$$

Dividing equation (36) by (37) leads to

$$
\frac{d \alpha}{d r}=-2 \alpha \overline{\tilde{u}^{2}} / r\left\{r^{2}\left(1-\alpha^{2}\right)+\overline{\tilde{u}^{2}}\right\}
$$

from which by integration for given initial conditions $\alpha_{0}=\alpha\left(\Phi_{0}\right)=1$ and $r_{0}=r\left(\Phi_{0}\right)$,

$$
r={\overline{\tilde{u}^{2}}}^{1 / 2} /\left\{2 \alpha\left(r_{0}^{2}+{\overline{\tilde{u}^{2}}}^{2} / 2 r_{0}{ }^{2}\right)-\alpha^{2}-1\right\}^{1 / 2} .
$$

Since

$$
u_{r}=\frac{d r}{d s}=\frac{d r}{d \Phi} \frac{d \Phi}{d s}
$$

and with equations (23) and (37)

$$
\overline{u_{r}}=\left\{r^{2}\left(1-\alpha^{2}\right)+\overline{\tilde{u}^{2}}\right\} / 2 \alpha r^{2}
$$

and further with equation (39)

$$
\overline{u_{r}}=\left(1+\overline{\tilde{u}^{2}} / 2 r_{0}{ }^{2}\right)-\alpha
$$

so that with $\alpha=\gamma-u_{r}$,

$$
\bar{\gamma}=1+\overline{\bar{u}^{2}} / 2 r_{0}{ }^{2} \text {. }
$$

\section{Speculations about cosmic ac- celeration}

The mean final energy of particles initially at rest thus exhibits an inverse proportionality to the square of the initial radial distance $r_{0}$ from the rotating magnetized star if $r_{\mathrm{B}}<r_{0}<r_{\mathrm{P}}$,

$$
\bar{\gamma} m c^{2}=\overline{\tilde{u}^{2}} m c^{2} / 2 r_{0}{ }^{2} .
$$

Adopting a constant source function for this region of space one is thereby lead to a differential energy spectrum which is characterized by an inverse proportionality to the $2.5^{\text {th }}$ power of energy in reasonable agreement with what is observed for high energy cosmic-ray particles. Pursuing this line of thought by further postulating an outer injection mechanism (Thielheim 1986) by which neutral particles from the interstellar medium enter into the given region of acceleration $r_{\mathrm{B}}<r_{0}<r_{\mathrm{P}}$ to be ionized there and to generate a constant source function of charged particles, the chemical composition of the latter can be expected to resemble the chemical composition of the interstellar medium, which is also in reasonable agreement with observations of high energy cosmic-ray particles. 